\title{
A Novel Regional Cloud Digital Library Network Based on Mobile Ad Hoc Networks
}

\author{
Zhiming Zhang ${ }^{1}$ and Wei Zhang ${ }^{2, *}$ \\ ${ }^{1}$ Department of Information Engineering, Engineering University of CAPF, China \\ $4406875 @ q q . \mathrm{com}$ \\ ${ }^{2}$ Refree of The 210th Institute of the Sixth Academy of CASIC, China \\ zwzy0717@163.com
}

\begin{abstract}
At present the digital library has entered into the period of cloud computing. The cloud digital library is a kind of virtual library, which is built upon Internet, and uses the cloud computing technology to provide services for readers. For the status and developing trend of cloud digital libraries, a regional cloud digital library network based on the mobile ad hoc network is proposed, the network architecture is designed, and its performance is evaluated by network simulation software NS-2. The result shows that the performance of the network is good, and it is feasible. The research result in the paper is valuable for the study and application of cloud digital libraries.
\end{abstract}

Keywords: cloud digital library, cloud computing, mobile Ad hoc network, region network architecture.

\section{Introduction}

The cloud computing technology is one of the most important technologies in information technology domain these years. In 2009, cloud computing was defined by National Institute of Standards and Technology (NIST) as a model in which networks were used to provide rapid and convenient services for a series of shared computer resources, nonetheless the administration cost in demand and suppliers' interaction cost are minimum 1. It leads the development of industry and society informatization, along with the popularity of Internet of things and mobile Internet; the permeation of cloud computing in various industries was becoming increasingly apparent 2. After experiencing the period of Internet, grid mesh and Web2.0 by sequence, digital library is entering into the period of "cloud computing". When using cloud computing, library services can reduce costs and improve efficiency greatly, according with the library's development needs. The largest organization for library cooperation in the world, Online Computer Library Center (OCLC) has already used cloud computer technology to establish a cloud digital library named OCLC Worldcat to provide services for readers in different countries 3. China Academic Library \& Information System (CALIS) is being constructed in the third stage, planning for building multilevel

\footnotetext{
* Corresponding author.
} 
sharing center using cloud computing technology and realizing the localized and low cost college digital libraries 4 .

During the past few years, some researchers have already investigated the theory and application of cloud digital library. Ref 5 surveys the advance on basic theory of cloud libraries, and points out the study on theory and application of cloud library includes five phases, i.e., consumers, resources, cloud services, cloud platform and cloud library administrators. Taking the cloud digital library in Shanxi University of Finance and Economics for example, ref 6 discusses the basic architecture and function of the cloud digital library, and explores the approaches and methods by which traditional libraries enter into the cloud digital libraries. Ref 7 proposes that the application of the cloud computing technology in libraries includes three developing phases, i.e., digital library, regional cloud library and total cloud library, and analyzes the transforming direction of traditional library and the challenges of the development of cloud libraries. Ref 8 proposes a novel service-oriented and layered regional cloud library, designs the architecture, which includes consumer layer, access layer, application portal layer, application layer, supporting tool layer, basic technology layer and cloud resource layer. However, in the above references, the authors only discuss the theory and application of the cloud digital library, or design the system model, without further simulation of the performance of the system model.

Based on the former work, the theory and application of the cloud digital library are further studied in the paper. Firstly, the concept and architecture of the cloud digital library is overviewed. Then, for the status and developing trend of cloud digital libraries, a regional cloud digital library network based on MANET is proposed, the network architecture is designed, and its performance is evaluated by network simulation software NS-2. The result shows that the performance of the network is good, and it is feasible. The research result in the paper is valuable for the study and application of cloud digital libraries.

\section{Overview of the Cloud Digital Library}

\subsection{Concept of the Cloud Digital Library}

Cloud digital library can be defined as a virtualization library based on Internet and providing all kinds of services for readers using the cloud computing technology. In other words, cloud digital library is library facility and service constructed by the cloud computing technology. The cloud digital library integrates the digital resources in several libraries by a cluster of parallel computers in a large-scale library. Thus, the cloud digital library can search resources and process data rapidly and conveniently, and can be accessed by users on demand. In the traditional library, different libraries cannot share each resource due to the disparity of every system. Whereas the cloud digital library can expand information services, change the library service mode, meet the personalized need of users, and thus bring vast opportunity to its development 6 .

\subsection{Advantages of the Cloud Digital Library}

The cloud digital library can not only avoid repeated construction to achieve full sharing of the resources, but also improve network performance and service efficiency greatly. Specifically, the advantages are as followed 9: 
(1) Reduce construction cost and improve operating efficiency. On the basis of cloud computing, the general medium or small scale libraries will not need to spend a lot of money on expensive hardware. They only need to construct their own cloud computing or ask the providers to do the construction, and the software could be upgraded and maintained online by providers. At the same time, the backward devices in library could still be fully used, such as to do the simple I/0 interactive computing. Also, we are no longer to worry about problems like data loss, computer virus or server being down, for there are millions of severs in "cloud". Therefore, if one sever goes wrong, the others could continue to work instantly, thus providing the most reliable and safest data storage center to the libraries.

(2) Bring down investment on repeated construction and realize resource sharing. The library which apply cloud computing could co-construct the information commons to share the information and resources with each other. All the digital library resources in the world could be gathered into the storage server of "cloud". The library administrator only needs to administrate, classify the resources and set out the matching visiting rules, and as long as following the rules, the users can obtain the digital resources from every corner of the world simply by entering the key word. The information resources could be fully shared in this case.

(3) Offer customized and personalized service. The current digital library for users could neither allocate resources according to the users' demands, nor provide individualized services. To provide resources according to the needs, and to charge per amount of usage are the outstanding merits of cloud computing. On the basis of cloud computing, the digital libraries will develop in a more personalized, liberalized and diversified direction. Every user could use the applications and digital resources gathered in the "cloud" to construct his personal digital library.

\subsection{Architecture of the Cloud Digital Library}

At present the architecture of cloud computing can be classified into three categories 10: SaaS (Software as a Service), PaaS (Platform as a Service) and IaaS (Infrastructure as a Service). SaaS is a method of supplying complete applications for the Internet as a service, such as Google Docs, Gmail and Salesforce.com 11. To develop and deploy custom applications, PaaS provides a platform, such as Google App Engine 12 and Microsoft Windows Azure 13. IaaS is a way of providing storage and elastic computing resources on demand, such as Amazon's Simple Storage Service (S3) 14, Elastic Compute Cloud (EC2) 15, and several open source implementations, for example, Eucalyptus 16 and OpenStack 17.

The architecture of the cloud digital library is shown in figure 1. According to the status of libraries and the service provided by cloud computing, its architecture can be generally classified into five layers, i.e., application layer, platform layer, data layer, hardware virtualization layer and infrastructure layer. Every layer is consisted of the corresponding cloud computing service, and can provide the service that the digital library can provide. For example, SaaS is used to constitute the application software, such as Platform for Library Content Selection, Automated Management System; PaaS 


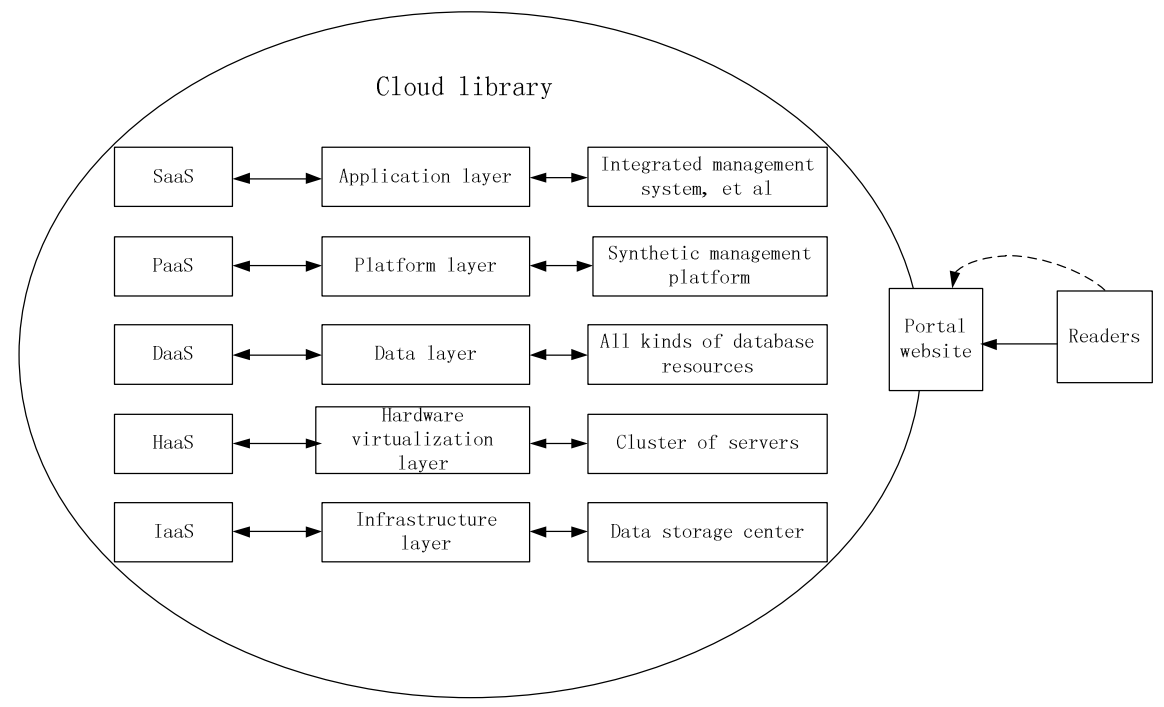

Fig. 1. Architecture of regional cloud digital library

is used to develop application service platform, and provides database services of the cloud digital library; Data as a Service (DaaS) is used to integrate the literatures in every library; Hardware as a Service (HaaS) is used to form the cluster of servers, owning the effective ability of flexible computing; IaaS is used to construct the storage and data center segment of the cloud digital library. Finally, the cloud digital library based on Internet is established. On the other hand, the portal website is created, and readers can enjoy the services provided by the cloud digital library 18 .

\section{Regional Cloud Digital Library Network Based on MANET}

\subsection{Concept of Regional Cloud Digital Library}

The construction and development of cloud digital libraries is a progressive process. The cloud digital library will develop gradually with the progress of the cloud computing-related technology and philosophy, and transit from the regional cloud digital library to the cloud digital library totally based on Internet gradually. At present, cloud computing technology is in the stage of preliminary research and application. Therefore, regional readers need-oriented networking services based on Internet are suitable to be carried on, and the regional cloud digital library can be constructed.

The regional cloud digital library is based on a digital library whose construction and service are senior in a certain region. Through the synthetic integration of technology, resource and service, the efficient software, hardware and administration platform of digital resource in digital libraries are established to provide every service of cloud digital libraries for the users in the region. The main function of the regional cloud digital library includes: (1) to provide literature-related service for the users in the region; (2) to provide software and hardware platform of digital libraries for other 
institutes in the region; (3) to provide literature, software and hardware platform, and administration integrated service for the users in the region 8.

\subsection{Architecture of Regional Cloud Digital Library Network Based on MANET}

The user network of the regional cloud digital library in the paper adopts Mobile Ad hoc Network (MANET). MANET is a kind of mobile wireless network, which is consisted of mobile nodes and does not rely on the network infrastructure. In MANET, nodes exchange data by their wireless sending and receiving equipments. When nodes are beyond their communication range, the multi-hop communication will be accomplished by the relay of other nodes. In the paper, MANET is used in the cloud digital library to making full use of advantages of flexibility and high efficiency.

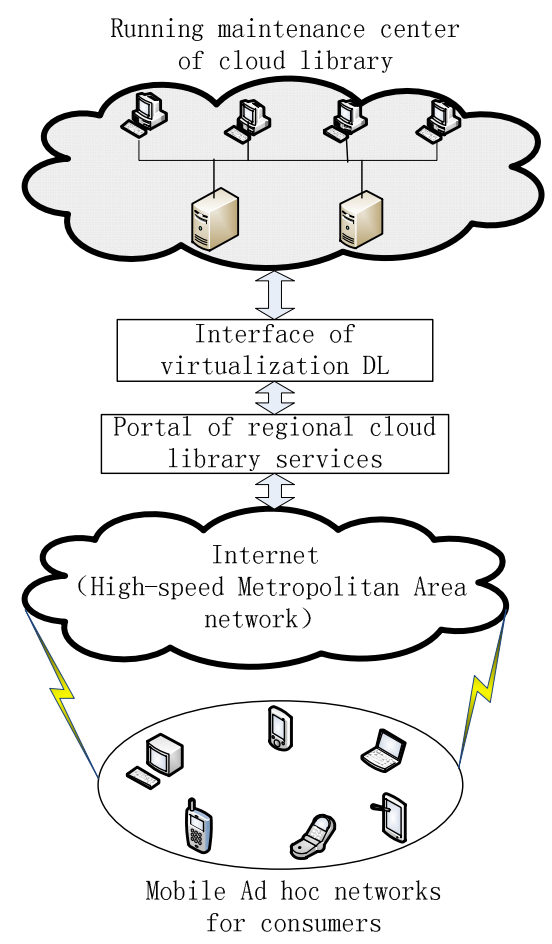

Fig. 2. Architecture of regional cloud digital library network based on MANET

The architecture of the regional cloud digital library network based on MANET is shown in figure 2. It can be divided into five layers, i.e., consumer MANET layer, Internet or high-speed MAN layer, portal website access layer, virtualization DL interface layer, and running maintenance center layer. The basic workflow of the network is: (1) the service portal is formed through integration of relative resources by the regional cloud digital library, and the user in the region can access the portal 
website through MANET using all kinds of network terminal; (2) Interface of virtualization DL adopts Service-Oriented-Architecture (SOA) and provides network service interface for portal website access layer; (3) the virtualization services of hardware, software and resources are realized through the virtualization technology of cloud computing by running maintenance center of the region cloud digital library, and the normal working of the network can be ensured.

Because of the security threat in data storage, reliability of cloud platforms and sustainability of services, user authority management, and virtualization, et al, faced by cloud digital libraries 19, the network we proposed in the paper adopts the scheme of static data encryption to manage the core data in cloud storage areas, the encryption and digital signature technologies in data's storage and transmission process, and Privilege Management Infrastructure (PMI) to control the users' authority for resources to ensure the security the data and services in the network.

\subsection{Simulation and Analysis}

Since MANET for consumers is the bottom layer of the regional cloud digital library network based on MANET proposed in the paper, the performance of the network will be simulated and evaluated preliminarily by network simulation platform NS-2. The network model is setup in NS-2.34, and the parameters are that the number of users is 10 to 50 , every node moves with the speed of $10 \mathrm{~m} / \mathrm{s}$, the size of simulation scenario is $5000 \times 5000 \mathrm{~m} 2$, the duration of simulation is $10 \mathrm{~min}$, and the routing and MAC protocol are DSR and IEEE 802.11 respectively. The simulation parameters and values are shown concretely in table 1 .

Table 1. Simulation parameters and values

\begin{tabular}{cccc}
\hline Parameters & Values & Parameters & Values \\
\hline Simulation area & $5000 \times 5000 \mathrm{~m}$ & Channel type & $\begin{array}{c}\text { Wireless } \\
\text { Channel }\end{array}$ \\
Communication range & $300 \mathrm{~m}$ & MAC & IEEE 802.11 \\
Routing protocol & DSR & $\begin{array}{c}\text { Queue type } \\
\text { Flow type }\end{array}$ & PriQueue \\
Maximum moving speed & CBR & $\begin{array}{c}\text { Simulation } \\
\text { time }\end{array}$ & $600 \mathrm{~s}$ \\
Antenna type & $10 \mathrm{~m} / \mathrm{s}$ & $\begin{array}{c}\text { Queue length } \\
\text { Channel } \\
\text { capacity }\end{array}$ & 50 \\
Propagation & Omni-Antenn & $100 \mathrm{Mps}$ \\
TwoRay-Gro & Data rate & $50 \mathrm{Mbps}$ \\
No. of users \& Maximal No. of & und & 10 \& 3, 20 \& 6, 30 \&9, 40 \& 12, 50 \& 15 \\
connection & &
\end{tabular}

After simulation, the packets delivery rate, average end-to-end delay, and route costs of the network with the number of the users in MANET are calculated, which are 
shown in figure 3 to 5 . From figure 3 to 5 , it can be found that every performance indicator of the network is good enough to meet the need of users to the cloud digital library. On the other hand, simulations show that with the increase of the number of users in MANET, the packets delivery rate decreases, the average end-to-end delay and route costs rise, which indicate the worsening of performance of the network. The reason is that when the number of users increases, the total traffic volume of the network increases, while the bandwidth of the network does not change. Therefore, the packet loss rate, end-to-end delay and route costs increase at the same time.

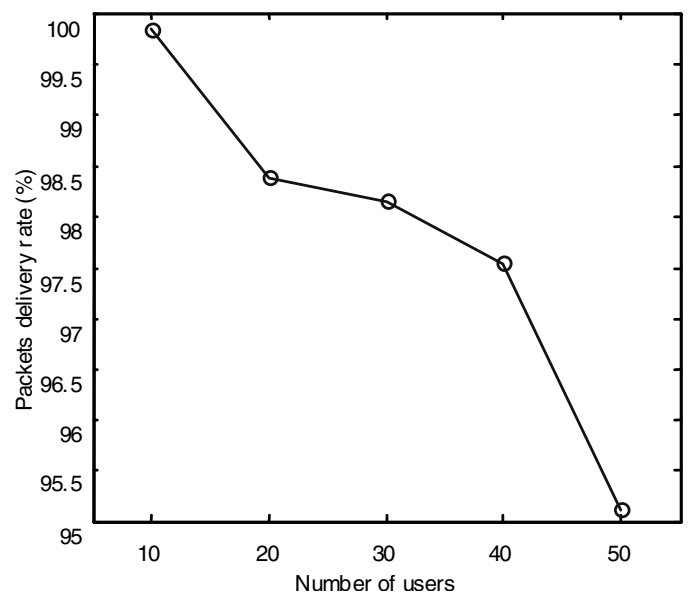

Fig. 3. Packets delivery rate of the network

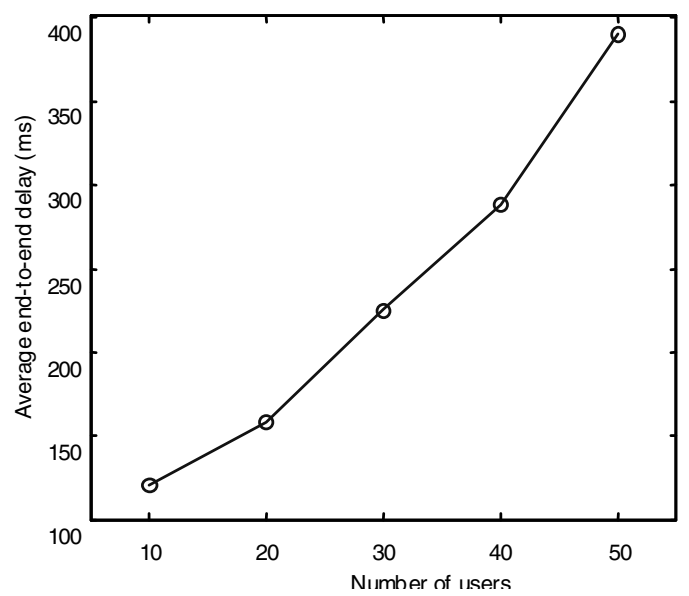

Fig. 4. Average end-to-end delay of the network 


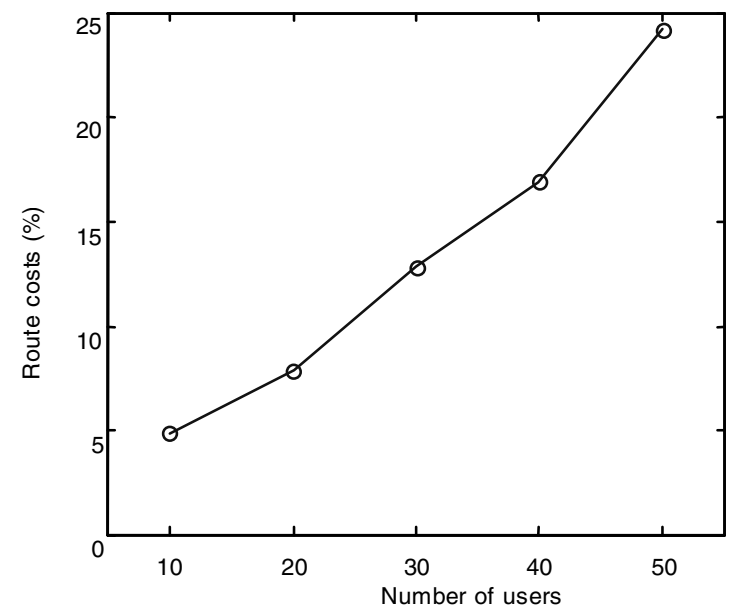

Fig. 5. Route costs of the network

\section{Conclusion}

For the status and developing trend of cloud digital libraries, a regional cloud digital library network based on MANET is proposed, the network architecture is designed, and its performance is evaluated by network simulation software NS-2. The result shows that the performance of the network is good, and it is feasible. The research result in the paper is valuable for the study and application of cloud digital libraries.

\section{References}

1. Mell, P., Grance, T.: The NIST Definition of Cloud Computing. National Institute of Standards and Technology [J/OL] (2009),

http://csrc.nist.gov/publications/nistpubs/800-145/ SP800-145.pdf

2. Meng, Q., Gong, C.: Research of Cloud Computing Security in Digital Library. In: 6th International Conference on Information Management, Innovation Management and Industrial Engineering, pp. 41-44 (2013)

3. Wen, Z.: The Inspiration of OCLC Worldcat Cloud Computering Digital Library on Government Information Services in China. Journal of Information Resources Management 4, 82-87 (2012)

4. Wang, W., Chen, L.: Model of Cloud Services Platform in CALIS Digital Library. Journal of Library in University 27(4), 13-18 (2009)

5. Zhang, X., Li, C., Qin, X.: A Study and Progress of the Basic Theory of Cloud Library. Library Tribune 32(9), 87-93 (2012)

6. Wang, H.: Architecture and Implementation of the "Cloud Library" Platform. Theory and Practice of Intelligence 33(10), 108-112 (2010) 
7. Hu, X.: Conception of Cloud Library. Theory and Practice of Intelligence 33(6), 29-32 (2010)

8. Hu, X., Shen, H., Zhang, Z.: Research on Construction of Regional Cloud Digital Library. Theory and Practice of Intelligence 34(2), 77-84 (2011)

9. Wei, M., Wang, F., Xu, X.: Development of Digital Libraries on the Basis of Cloud Computing. In: IEEE International Conference on Computer Science and Service System, p. $304(2012)$

10. Lu, W., Zheng, L., Shao, J., et al.: Digital Library Engine: Adapting Digital Library for Cloud Computing. In: IEEE Sixth International Conference on Cloud Computing, pp. 934-941 (2013)

11. Salesforce Customer Relationships Management (CRM) System, http: / /www.salesforce.com/

12. Google AppEngine, http://code.google.com/appengine

13. Windows Azure Platform, http: //www. microsoft.com/windowsazure/

14. Amazon Simple Storage Service, http: / / aws . amazon.com/s3/

15. Amazon Elastic Compute Cloud, http: / / aws . amazon. com/ec2 /

16. Nurmi, D., Wolski, R., Grzegorczyk, C., et al.: The Eucalyptus Open-Source Cloud-Computing System. In: CCGRID 2009 (2009)

17. OpenStack: The open source, open standards cloud, http: / / openstack .org

18. Vaquero, L.M., Rodero-Merino, L., Caceres, J., et al.: A Break in the Clouds: Towards a Cloud Definition. ACM SIGCOMM Computer Communication Review 39(1), 50-54 (2009)

19. Ma, X.-T., Chen, C.: Study on Cloud Computing Security Analysis and Management Strategy for Digital Library. Intelligence Science 29(8), 1186-1191 